\title{
From Mathematics to Software Engineering: Introducing Category Theory into the Computer Science Curriculum ${ }^{\star}$
}

\author{
Yujun Zheng ${ }^{1,2,3}$, Haihe $\mathrm{Shi}^{1,2}$, and Jinyun Xue ${ }^{1,2}$ \\ ${ }^{1}$ Institute of Software, Chinese Academy of Sciences, 100080 Beijing, China \\ yujun.zheng@computer.org \\ ${ }^{2}$ Coll. Computer Inf. \& Eng., Jiangxi Normal University, 330027 Nanchang, China \\ ${ }^{3}$ Graduate University of the Chinese Academy of Sciences, 100080 Beijing, China
}

\begin{abstract}
Category theory, with its increasing role in computer science, has proved useful in the investigation of programming languages and other theoretical aspects of software engineering. As a bridge-building exercise, we introduce the category theory course into the computer science curriculum, the purpose of which includes building a unified framework to generalize the discrete structures of mathematical courses and providing an environment for formulating concepts of other software engineering courses. We develop a teaching schema, summarize our experiences, and give some suggestions for pedagogical techniques for the course.
\end{abstract}

\section{Introduction}

Category theory has developed rapidly, and its influence is being felt in many parts of computer science including the design and implementation of programming languages, models of concurrency, type theory, polymorphism, specification language, constructive logic, automata theory, and the development of algorithms [1]. Nevertheless, as a relatively young branch of pure mathematics, category theory is mainly incorporated into the mathematical curriculum for postgraduate education in a handful of universities, and it is widely supposed that the subject could only be understood by those with nontrivial facts in algebraic topology.

The motivation for this paper derives from our experience in introducing the category theory course for graduates in computer science over the last three years. Its aim is not to work through the mathematical derivations and proofs of the abstract theory, but to provide a high-level, categorial understanding of mathematical constructions in various aspects of computer science and software engineering. The key role of the course in the curriculum can be divided into two parts:

* Supported in part by grants from NNSF (No. 60573080) and NGFR 973 Program (No. 2003CCA02800) of China. 
1. Building a sound, unified framework that generalizes the structures learned from the preceding mathematical courses such as discrete mathematics, set theory and graph theory.

2. Providing a mathematical environment to formulate uniform definitions of the preceding, following, or concurrent software engineering courses including data structures and algorithms, object-oriented design, programming languages and formal methods.

The above two parts are described respectively in Section 2 and Section 3. Section 4 proposes some strategies and suggestions for teaching the category theory course, and Section 5 concludes with discussion.

\section{Generalization of Mathematical Structures}

The introductory category theory course usually starts with a review of discrete mathematics, from which students have learned a number of fundamental discrete structures and gained a basic knowledge of the mathematical foundations for computer science. First, elementary definitions in category theory should be introduced in terms of ordinary set theory: collections are sets, operations are set-theoretic functions, and equalities are set-theoretic identities. Second, the fundamental notions of category theory is that of a monoid: a set with a binary operation of multiplication that is associative and that has a unit; a category itself can be regarded as a sort of generalized monoids [2]. In this way, students can quickly have a self-contained and structural understanding of categories.

Alternatively, category theory deals in an abstract way with structures, i.e., offers a highly formalized language for expressing general properties and analyzing relations between classes of mathematical structures. By presenting a variety of categories of familiar structures, we can give students a unified, categorial understanding of mathematical structures. Those typical categories include:

- $\mathbf{N}$ with natural numbers as objects and ordering $n \rightarrow n+1$ as morphisms.

- Rel with sets as objects and relations as morphisms.

- Set with sets as objects and total functions between sets as morphisms.

- Poset with pre-ordered sets as objects and monotonic functions as morphisms. A pre-order is a category where there is at most one arrow between any two objects.

- Mon with monoids as objects and morphisms of monoids as (categorial) morphisms. A monoid is a category with one object.

- Grp with groups as objects with morphisms of groups. A group is a category with one object in which each arrow has an inverse under composition.

- Ab with additive abelian groups as objects with morphisms of such.

- Rng with rings as objects with the ring morphisms preserving units.

- K-Mod with modules over the communicative ring $K$ as objects and $K$ linear maps as morphisms.

- Top with topological spaces as objects and continuous functions as morphisms. 
Next, we can show how to use the categorial approach to achieve the generality of various concepts; that is, many different formalisms and structures may be proposed for what is essentially the same concept [3] in the language of categories. For example, the coproduct of any two objects exists in many categories, where it has various names as follows:

- Set: disjoint union of sets.

- Grp: free product.

- Ab: direct sum $A \oplus B$.

- Top: disjoint union of topological spaces.

\section{Theoretical Standardization of Software Engineering Methods}

\subsection{Data Structures}

After a generalization of mathematical structures, we should move towards data structures frequently used in computer programming. While the traditional set theory generally considers concrete implementations, category theory describes objects more as abstract data types, or specifications that are independent of particular implementations. Category localization semantics for specifications by Goguen [4] need to be introduced, followed by the exploration of category theory as a framework for designing, understanding, manipulating and evolving data structures. For example, specifications of data structures Preorder and $A n$ tisymmetry can be defined as follows:

\section{Preorder: sort $E$}

$$
\begin{aligned}
& \text { op } \leq: E, E \rightarrow \text { Boolean } \\
& \text { axiom reflexivity: } x \leq x \\
& \text { axiom transitivity: } x \leq y \wedge y \leq z \Rightarrow x \leq z
\end{aligned}
$$

\section{Antisymmetry: sort $E$}

$$
\begin{aligned}
& \text { op } \leq: E, E \rightarrow \text { Boolean } \\
& \text { axiom antisymmetry: } x \leq y \wedge y \leq x \Rightarrow x=z
\end{aligned}
$$

An initial object in the category $S P E C$ can be worked out as a specification that shares the properties of above two specification:

BinaryRelation: sort $E$

$$
\text { op } \leq: E, E \rightarrow \text { Boolean }
$$

\footnotetext{
${ }^{1}$ In brief, a specification is the finite presentation of a theory, and a signature provides the vocabulary of a specification; $S P E C$ is a category with specifications as objects.
} 
By constructing the morphisms $f:$ BinaryRelation $\rightarrow$ Preorder and $g$ : BinaryRelation $\rightarrow$ Antisymmetry and computing the colimit of $f$ and $g$, the composition of Preorder and Antisymmetry can be mechanically generated:

Partial Order: sort $E$

$$
\begin{aligned}
& \text { op } \leq: E, E \rightarrow \text { Boolean } \\
& \text { axiom reflexivity: } x \leq x \\
& \text { axiom transitivity: } x \leq y \wedge y \leq z \Rightarrow x \leq z \\
& \text { axiom antisymmetry: } x \leq y \wedge y \leq x \Rightarrow x=z
\end{aligned}
$$

In a word, under the categorial framework, the basic principle to specify a system is to build the structures for each component separately, and then use the colimit operation to compose these specifications [5]. This also provides an oriented direction from structured design to component-based software engineering (CBSE).

\subsection{Programming Languages}

Based on the categorial understanding of data structures that constitute the theoretic background of language design, we can lead students to relate category theory with widely used programming methodologies and to investigate mathematical semantics of programming languages.

A simplest example is given by (deductive) logic programming languages as categories, in which propositions are objects and proofs are morphisms. Such categories can be easily constructed and understood in the presence of the identical proof: $a \rightarrow a$ and the associative compositions of proofs: $(a \rightarrow b) \wedge(b \rightarrow$ $c) \Rightarrow(a \rightarrow c)$.

We can also show how to construct categories corresponding with functional programming languages. Consider a simple language with four primitive types Boolean, Integer, Double, and Element, the corresponding category contains primitive types as objects and constants and built-in operations as morphisms, as illustrated in Fig. 1.

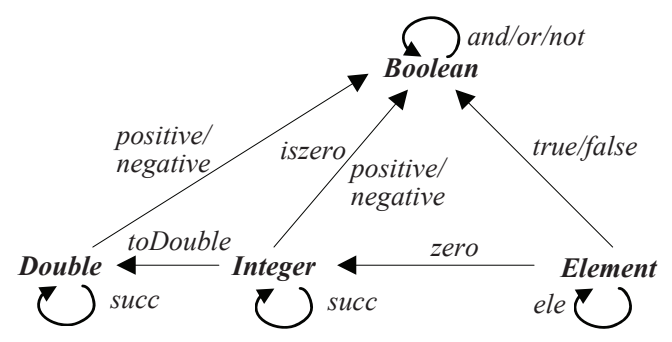

Fig. 1. A category representing a funtional programming language

Given the facility of category theory to defined more structured categories out of simper ones, many other features of modern functional languages, including formal and concrete parameters of functions, parameterized data types, 
polymorphic functions and recursive functions, can be addressed by stepwise introducing more properties of category theory, such as principle morphisms, exponentiation, and natural transformation [1. Afterwards, we can go deep into extra topics including type-free and second order lambda-calculus.

\subsection{Object-Oriented Design}

As mentioned above, category theory studies "objects" and "morphisms" between them: any immediate access to the internal structure of objects is prevented, and all the concepts must be defined by their relations with other objects. This is quite similar to ideas of object-oriented design. Therefore we can guide students to construct object-oriented categories step by step (the process of which abstracts the transition from structured programming to object-oriented programming). Such a underlying categorial framework for object-oriented design can be summarized as follows:

- $\boldsymbol{O B J} \boldsymbol{S} \boldsymbol{S I G}$ is a category with object signatures $\theta=<\Sigma, A, \Gamma>$ (where $\Sigma=\left\langle S, \Omega>\right.$ is a universe signature, $A$ is an $S^{*} \times S$-indexed family of attribute symbols, and $\Gamma$ is an $S^{*}$-indexed family of action symbols) as objects, and an object signature morphism is a consistent mapping from one signature to another.

- $\boldsymbol{O B} \boldsymbol{J}-\boldsymbol{S P E C}$ is a category with object specifications $O S P=<\theta, \Phi>$ (where $\Phi$ is a finite set of $\theta$-axioms) as objects and a morphism between specification $<\theta_{1}, \Phi_{1}>$ and specification $\left\langle\theta_{2}, \Phi_{2}>\right.$ is a mapping of signature $\theta_{1}$ into signature $\theta_{2}$ such that all the axioms in $\Phi_{1}$ are translated to theorems in $\Phi_{2}$.

- Let $D_{1}, D_{2} \ldots D_{n}$ be $\omega$-diagrams in $O B J-S P E C$ and $C O L_{i}$ be colimits for $D_{i}(i=1,2, \ldots n)$, then $\boldsymbol{C L} \boldsymbol{S}-\boldsymbol{S P E} \boldsymbol{C}$ is a category with $C O L_{i}$ as objects, and a class morphism between $C O L_{1}$ and $C O L_{2}$ is the colimit of all morphisms in $O B J-S P E C$ that between an object in $D_{1}$ and an object in $D_{2}$.

- $\boldsymbol{I}-\boldsymbol{F R} \boldsymbol{M}-\boldsymbol{S P E} \boldsymbol{C}$ is a category with diagrams in $O B J-S P E C$ as objects, and a morphism between two (implemented) object frameworks, namely $I-F R M_{1}$ and $I-F R M_{2}$, is the colimit of morphisms in $O B J-S P E C$ that between an object of $I-F R M_{1}$ and an object of $I-F R M_{2}$.

Morphisms in $O B J-S P E C$ may represent object relationships such as message connection, whole-part, generalization/specialization, etc., which are upgraded to morphisms in $C L S-S P E C$ including association, aggregation, and inheritance 6]; functors from $C L S-S P E C$ to $O B J-S P E C$ can be treated syntactically as instantiations or refinements, and functors from $O B J-S P E C$ to I-FRM-SPEC as compositions. In a similar way, categories $M-C L S-S P E C$ (of meta-classes), $M M$ $C L S$-SPEC (of meta-meta-classes), FRM-SPEC (of class frameworks), $M-F R M$ $S P E C$ (of meta-frameworks), and $M M-F R M-S P E C$ (of meta-meta-frameworks) can also be constructed [7]. As illustrated in Fig. 2, the left refinement process of class-based specifications is brought to the right refinement process of frameworkbased specifications through compositions at different levels of abstraction. 


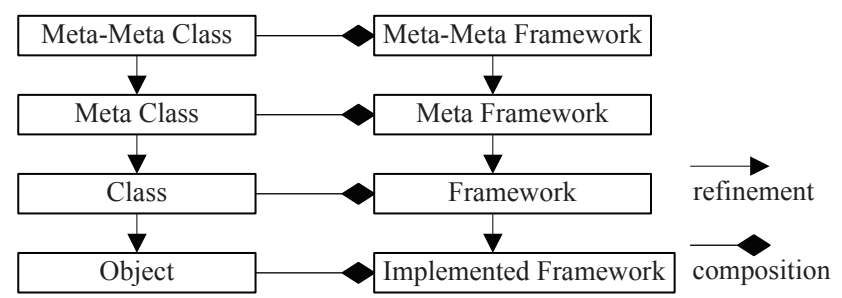

Fig. 2. Constructing object-oriented categories

As a typical paradigm of object-oriented design framework, design patterns can be employed to illustrate the application of theory-based approach in largescale and mechanizable software reuse [8. In detail, a design pattern is a (category) object in $M-F R M-S P E C$, and one of its refinement pathes to I-FRM-SPEC can be implemented rigorously and saved; when encountering another problem within the same pattern context, we can utilize the "standard" refinement to generate a new implementation via category theoretic computations. For example, suppose the Observer pattern [9] has been refined into an object framework (i.e., an executable program), namely $F R M_{1}$, where two observers DigitalClock and AnalogClock query the subject Timer to synchronize their time with the Time's state. As shown in Fig. 3, to work out a new framework (namely $F R M_{2}$ ) representing the underlying dataset in separate forms of interface, we just construct a functor $H_{1}{ }^{2}$ from $F R M_{1}$ and generate the program $I-F R M_{2}$ without refining $G_{2} \circ G_{1}$ manually: $G_{1}$ is the composition $H_{1} \circ F_{1}$, while $G_{2}$ and $H_{2}$ are obtained by computing the colimit of $H_{1}$ and $F_{2}$.

\section{Guiding Strategies and Suggestions}

Arising in algebraic topology as a way to explain in what sense the passages from geometry to algebra are "natural" in the sense of reflecting underlying geometric reality [10, category theory would be considered esoteric by many students in computer science. Based on our lessons and experiences, we propose the following strategies and suggestions for improving the pedagogical effectiveness of the course:

- Utilizing diagrams. Diagrams are an important tool in the practice of category theory, especially for describing equational reasoning. From beginning, we should help students learn how to go back and forth from diagrams to equations, and use diagrams as possible for stating and proving properties of categorial constructions.

- Informal explanation. Many category theoretic formalizations are too abstract and heavily notational for students. To avoid the expense of devious

${ }^{2}$ Which is the colimit of the four morphisms: $f_{0} \rightarrow g_{0} ; f_{1} \rightarrow g_{1} ; \operatorname{coequalizer}\left(f_{2}, f_{3}\right) \rightarrow$ coequalizer $\left(g_{2}, g_{3}, g_{4}\right)$; coequalizer $\left(f_{4}, f_{5}\right) \rightarrow$ coequalizer $\left(g_{5}, g_{6}, g_{7}\right)$. 


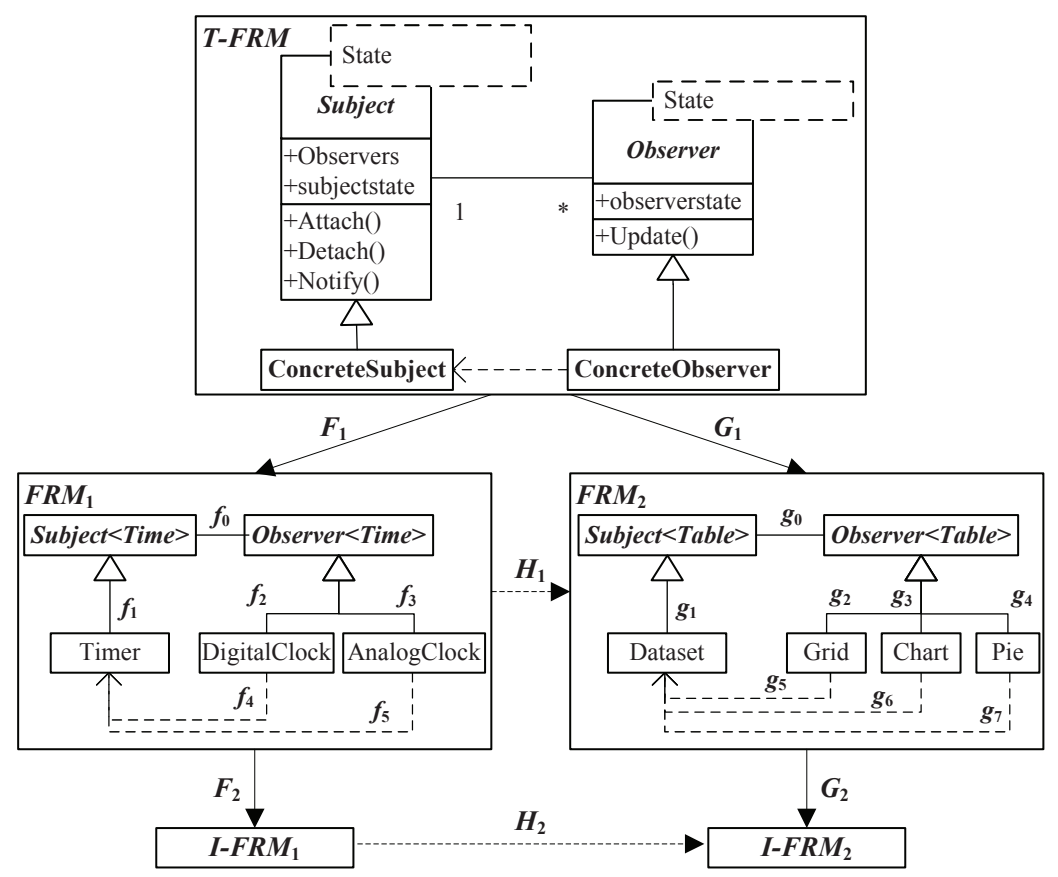

Fig. 3. Mechanization of deign patterns via category theoretic computations

circumlocutions, most lectures should begin with a clear definition, prefaced with an informal explanation of the construction, and followed by accessible examples illustrating the methodological connection and applications in computer science.

- Avoid misleading. Connection between category theory and set theory is helpful to introduce many categorial concepts, but we should be careful because sometimes it can be misleading. For example, a morphism that is both epic (surjective) and monic (injective) does not need to be isomorphic, and in a Cartesian closed category a reflexive object $a$ is given by $a^{a}<a$, which are clearly impossible in ordinary set theory.

- Guide to computation. For students in computer science, we should give more emphasis on the presentation of ideas and methods than mathematical notions and proofs, exhibit the intriguing advantages of formalizing and mechanizing computations in categorial principles, and help students learn to model and reason categorically.

- Orientation to specific research. Within limited credit hours, it is impossible to work through the constructions, derivations and proofs of all important propositions and theorems. Category theory is, by and large, a self-contained discipline and one can go a long way without mentioning the traditional mathematical examples [11]. The course is just a doorstep, and 
its main purpose is to give a familiarity with the category theory and make the students prepared to approach further researches applying the theory to various aspects of computer science.

\section{Conclusions}

As a "theory of functions", category theory offers a highly formalized language which is suitable for focusing concerns on various areas of computer science and software engineering. The paper summarizes our efforts and experiences in introducing the category theory course into the computer science curriculum, the role of which includes building a unified framework that generalizes the structures of preceding mathematical courses and providing a mathematical environment to formulate uniform definitions of the other software engineering courses. The main purpose is not to work through the mathematical derivations and proofs, but to give students a structural understanding of categorial constructions and improve their mathematic literacy for further researches in computer science.

\section{References}

1. Pierce, B.C.: Basic Category Theory for Computer Scientists. MIT Press, Cambridge, London (1991)

2. Saunders, M.L.: Categories for the Working Mathematician. Springer-Verlag, New York (1998)

3. Asperti, A and Longo, G.: Categories, Types and Structures: an introduction to category theory for the working computer scientist. MIT Press, Cambridge (1991)

4. Goguen, J.A.: A Categorical Manifesto. Mathematical Structures in Computer Sciences, vol. 1 (1991) 49-67

5. Wiels, V. and Easterbrook, S.: Management of Evolving Specifications Using Category Theory. In Proceedings of 13th IEEE Conference on Automated Software Engineering, Hawaii (1998) 12-21

6. Kung, D.C., Gao, J., Hsia, P., Toyoshima, Y., and Chen, C.: On Regression Testing of Object-Oriented Programs. Journal of Object-Oriented Programming, vol. 8, no. 2 (1995) 51-65

7. Zheng, Y.J., Xue, J.Y., and Liu,W.B.: Object-Oriented Specification Composition and Refinement via Category Theoretic Computations. In Proceedings of 3rd International Conference on Theory and Applications of Models of Computation, Beijing, China, 2006. Lecture Notes in Computer Science, vol. 3959 (2006) 601-610

8. Zheng, Y.J., Shi, H.H., and Xue, J.Y.: Formalization and Mechanization of Design Patterns. In Proceedings of 1st International Conference on Computer Science and Education, Xiamen, China (2006) 892-897

9. Gamma, E., Helm, R., Johnson, R., and Vlissides J.: Design Patterns: Elements of Reusable Object-Oriented Systems. Addison-Wesley, Reading MA (1995)

10. Rydeheard, D.E. and Burstall, R.M.: Computational Category Theory. Prentice Hall, New York (1988)

11. Turi, D.: Category Theory Lecture Notes. Laboratory for Foundations of Computer Science, University of Edinburgh (2001) 